\title{
Activated protein $C$ response and cardiovascular risk factors in the elderly - The Rotterdam Study
}

\author{
M.L. Bots', J.G. van der Bom ${ }^{1,2}$, F. Haverkate' ${ }^{2}$ P. Meijer', A. Hofman', D.E. Grobbee', \\ C. Kluft ${ }^{2}$
}

'Department of Epidemiology \& Biostatistics, Erasmus University Medical School, Rotterdam, The Netherlands.

${ }^{2}$ Gaubius Laboratory, TNO-PG, Leiden, The Netherlands.

Summary Background and objective A low activated protein $C$ (APC) response increases the risk of venous thrombosis, and possibly arterial disease. Apart from the factor $V$ Leiden mutation, other factors may determine the level of APC response. In most studies on the association with arterial disease, cardiovascular risk factors were not appropriately controlled for as possible confounders. We studied the association of APC response to cardiovascular risk factors in a sample of the general population.

Methods The Rotterdam Study is a cohort study among 7983 subjects aged 55 years or over, living in the Ommoord suburb in Rotterdam, The Netherlands. APC response was measured in 928 subjects as part of two case-control studies (subjects with and without symptomatic cardiovascular disease (CVD) and with and without dementia). APC response was

determined in double centrifuged platelet poor plasma. Those on anticoagulants were excluded. Linear regression analyses were done in age strata $(55-64,65-74,75-84, \geq 85)$, with adjustment for age, sex, CVD, and dementia.

Results APC response was lower in women than in men (difference $0.56(p<0.01))$. In men, APC response decreased with age $(0.15$ per decade $(p=0.02))$. In women, a non-significant rise was seen $(0.05$ per decade $(p=0.27))$. Associations with cardiovascular risk factors differed by age, not gender. In those aged 55-64 years inverse associations were found with total cholesterol $(0.14$ decrease per $1 \mathrm{mmol} / \mathrm{l}$ increase, $p=0.03)$ and diastolic pressure $(0.12$ decrease per $10 \mathrm{mmHg}$ increase, $p=0.06)$. In those aged $\geq 85$ years a positive association was seen with smoking $(0.76$ increase, $p=0.01)$. No associations were found with systolic pressure and body mass index.

Conclusion These findings suggest that in the elderly age, gender, serum lipids, diastolic pressure and smoking may influence APC response, the extent of which may differ by age. These factors should be allowed for in studies on the association between APC response and arterial disease.

\section{INTRODUCTION}

A low response to activated protein $\mathrm{C}$ (APC) points towards a decreased ability of activated protein $\mathrm{C}$ to exert its natural anticoagulant properties. Consequently one may envision that a low APC response may lead to increased risk of venous thrombosis and possibly arterial disease. A low APC response has indeed been identified as a risk factor for venous thrombosis. ${ }^{1,2}$ Its relation with arterial disease remains, however, to be established. ${ }^{3,4}$ A low APC response has frequently been reported to be due to a single

Correspondence to: Michiel L. Bots, MD, PhD, Department of Epidemiology \& Biostatistics, Erasmus University Medical School, P.O. Box 1738, 3000 DR Rotterdam, The Netherlands. Tel. +31-10-4087489; Fax. +31-104365933 . base mutation of the factor $\mathrm{V}$ gene..$^{5-7}$ Consequently, the APC response test has been widely used as a screening test for the factor $\mathrm{V}$ Leiden mutation, in which an arbitrary cutoff point among control subjects was chosen to identify subjects 'resistant' to activated protein C. APC response is, however, a continuously distributed characteristic and, apart from the factor $\mathrm{V}$ Leiden mutation, other factors may determine the level of an APC response. ${ }^{8}$ In most studies on the association with arterial disease, APC response was dichotomized and established cardiovascular risk factors were not appropriately allowed for as possible confounders. Most studies were performed among young and middleaged subjects.

We evaluated the association between APC response and established cardiovascular disease risk factors in a sample of the general population, aged 55 years or over. 


\section{METHODS}

\section{Population}

The Rotterdam Study is a single centre prospective followup study of disease and disability in the elderly, described in detail elsewhere. ${ }^{9}$ Eligible participants included all residents 55 years and older living in the Rotterdam suburb of Ommoord, in the Netherlands. Baseline measurements were made from March 1990 to July 1993. Of the 10,275 eligible participants, 7,983 participated $(78 \%)$. The study has been approved by the Medical Ethics Committee of the Erasmus University and written informed consent was obtained from all participants.

The analyses presented here are based on 928 subjects that were part of two case-control studies. One case control study consisted of case-subjects, who were selected on the presence of either a myocardial infarction on the electrocardiogram $(n=92)$, history of angina pectoris $(n=123)$, a history of stroke $(n=48)$, a history of transient ischemic attack $(n=50)$ or presence of peripheral arterial disease $(n=112)$, and control subjects $(n=218)$, who were free from symptomatic cardiovascular disease, had a normal ECG and an ankle-to-brachial blood pressure index above $0.9 .^{4}$ The other case control study consisted of demented subjects $(n=99)$ and non-demented control subjects $(n=209)$. Some subjects were part of both casecontrol studies $(n=32)$. Control subjects for both casecontrol studies were randomly drawn from the Rotterdam study participants, frequency matched on age. Subjects on anticoagulant drugs were excluded.

\section{Cardiovascular risk factors}

A subject's smoking status, based on data obtained during a home interview, was classified as current, former, and never smoker. Data on other risk factors were collected at the Rotterdam Study research centre. Height and weight were measured and body mass index calculated $\left(\mathrm{kg} / \mathrm{m}^{2}\right)$. Sitting blood pressure was measured on the right upper arm with a random-zero sphygmomanometer using a 32 by 17 $\mathrm{cm}$ cuff. Diastolic blood pressure was recorded at the fifth phase. The average of two measurements obtained at one occasion, separated by a count of the pulse rate, was used in the analysis.

Blood sampling procedures and storage have been described elsewhere. ${ }^{10}$ In short, non-fasting blood was collected in tubes containing $0.129 \mathrm{M}$ sodium citrate. All tubes were stored on ice before and after blood sampling. Platelet poor plasma was obtained by a two stage centrifugation: firstly at $1600 \mathrm{~g}$, at $4^{\circ} \mathrm{C}$ for 10 minutes and after carefully transferring the plasma midlayer a second centrifugation was performed at $10000 \mathrm{~g}$, at $4{ }^{\circ} \mathrm{C}$ for 10 minutes. Plasma was immediately frozen in liquid nitrogen and stored at $-80^{\circ} \mathrm{C}$. Plasma from 30 apparently healthy volunteers was centrifuged for 30 minutes at $2000 \mathrm{~g}$, at $4^{\circ} \mathrm{C}$ and pooled to serve as reference plasma. The response of the plasma activated partial thromboplastin time (APTT) to APC was determined using the Coatest APC resistance test of Chromogenix, Mölndal, Sweden and expressed as the ratio of APTT with and without addition of APC. Assays were performed on a MLA Electra $1000 \mathrm{C}$. APC response in the pooled plasma was 3.27. Fibrinogen was determined using a nephelometric method. Factor VIII activity was determined using a chromogenic assay. Total cholesterol and high density lipoprotein (HDL) cholesterol were determined by an automated enzymatic procedure.

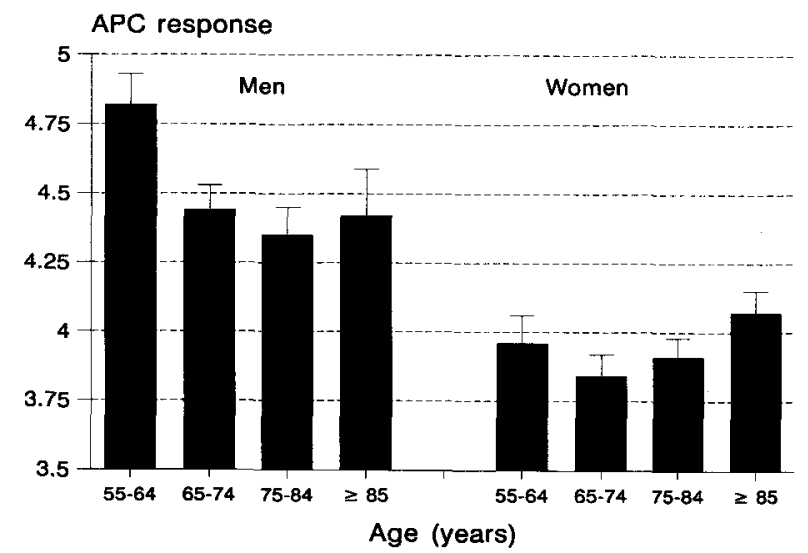

Fig. 1 APC response by age and gender.

\section{Data analysis}

A priori, multivariate linear regression analyses were performed in strata of age $(55-64,65-74,75-84, \geq 85)$ and gender (men, women). Results are adjusted for age, gender, symptomatic cardiovascular disease and dementia. APC resistance was defined as a APC response lower than 2.05, calculated as the (mean-2SD) based on the APC distribution in 312 subjects free from cardiovascular disease and dementia.

\section{RESULTS}

General characteristics are described in table 1. Figure 1 shows that APC response was lower in women than in men with a mean difference of $0.56(95 \% \mathrm{CI} 0.40,0.70)$. In men, APC response decreased with age $(0.15$ per decade, $(95 \%$ CI 0.03,0.27)). For women, a non significant increase with age was seen $(0.05$ per decade $(95 \%$ CI $0.03,0.13)$. The prevalence of APC resistance was $0.9 \%$ (95\% CI $0.1-2.1)$ in men and $1.5 \%(95 \% \mathrm{CI} 0.5-2.5)$ in women. 
Table 1 General characteristics of the study population.

\begin{tabular}{llllll}
\hline & \multicolumn{5}{c}{ Age (years) } \\
Characteristic & $55-64$ & $65-74$ & $75-84$ & $\geq 85$ & All \\
\hline & & & & & \\
Number & 196 & 260 & 292 & 180 & 928 \\
Age (arrows) & $61.0(2.7)$ & $70.0(2.7)$ & $79.8(2.9)$ & $88.8(3.0)$ & $74.8(10.0)$ \\
Women (\%) & 54.6 & 53.0 & 66.8 & 78.8 & 62.7 \\
Dementia (\%) & 0.5 & 6.1 & 19.1 & 26.6 & $13.0^{*}$ \\
Symptomatic CVD & 71.8 & 58.4 & 58.9 & 62.2 & $62.1^{*}$ \\
& & & & & \\
Systolic pressure $(\mathrm{mmHg})$ & $135(21)$ & $144(24)$ & $148(24)$ & $149(28)$ & $145(25)$ \\
Diastolic pressure $(\mathrm{mmHg})$ & $74(11)$ & $76(12)$ & $73(13)$ & $72(14)$ & $74(13)$ \\
Total cholesterol $(\mathrm{mmol} / \mathrm{l})$ & $6.8(1.1)$ & $6.6(1.2)$ & $6.4(1.2)$ & $6.1(1.4)$ & $6.5(1.2)$ \\
HDL cholesterol $(\mathrm{mmol} / \mathrm{l})$ & $1.3(0.4)$ & $1.3(0.4)$ & $1.3(0.4)$ & $1.4(0.3)$ & $1.3(0.4)$ \\
Body mass index $\left(\mathrm{kg} / \mathrm{m}^{2}\right)$ & $26.1(3.6)$ & $26.6(3.5)$ & $26.5(4.0)$ & $25.2(3.7)$ & $26.2(3.7)$ \\
Current smoking $(\%)$ & 28.5 & 19.2 & 12.2 & 12.2 & 17.6 \\
& & & & & \\
APC response & $4.36(1.1)$ & $4.13(1.0)$ & $4.05(1.0)$ & $4.13(1.2)$ & $4.15(1.1)$ \\
\hline
\end{tabular}

Values are proportions or means with standard deviations in parentheses. Results are unadjusted.

* Selection criterium for case-control study, from which the APC data are derived.

Table 2 Mean change in APC response (standard error) with one unit increase in a cardiovascular risk factor.

\begin{tabular}{|c|c|c|c|c|c|c|c|c|c|c|c|c|c|c|c|}
\hline \multirow{3}{*}{ Risk factor } & \multicolumn{11}{|c|}{ Age (years) } & \multirow{2}{*}{\multicolumn{4}{|c|}{ Total group }} \\
\hline & \multirow{2}{*}{$\begin{array}{l}55-64 \\
\text { Mean }\end{array}$} & \multirow[b]{2}{*}{ SE } & \multicolumn{3}{|c|}{$65-74$} & \multicolumn{3}{|c|}{$75-84$} & \multicolumn{3}{|c|}{$\geq 85$} & & & & \\
\hline & & & $p$ & Mean & SE & $p$ & Mean & SE & $p$ & Mean & SE & $p$ & Mean & SE & $p$ \\
\hline Systolic pressure $(10 \mathrm{mmHg})$ & 0.02 & $(0.04)$ & 0.65 & $0 . \overline{03}$ & $(0.03)$ & 0.32 & 0.01 & $(0.03)$ & 0.70 & 0.01 & $(0.03)$ & 0.97 & 0.01 & $(0.02)$ & 0.48 \\
\hline Diastolic press & -0.12 & $(0.06)$ & 0.06 & 0.06 & $(0.05)$ & 0.23 & 0.09 & $(0.05)$ & 0.04 & 0.04 & $(0.07)$ & 0.51 & 0.03 & $(0.03)$ & 0.29 \\
\hline Total cholesterol (1 & -0.14 & $(0.07)$ & 0.03 & 0.03 & $(0.05)$ & 0.57 & -0.02 & $(0.05)$ & 0.68 & -0.01 & $(0.10)$ & 0.88 & -0.05 & $0.03)$ & 0.12 \\
\hline HDL cholesterol $(1 \mathrm{mmol} / \mathrm{l})$ & -0.07 & $(0.22)$ & 0.75 & -0.08 & $(0.17)$ & 0.62 & -0.03 & $(0.17)$ & 0.85 & 0.26 & $(0.26)$ & 0.32 & -0.01 & $(0.10)$ & 0.88 \\
\hline Body & -0.11 & $(0.19)$ & 0.56 & 0.08 & $(0.18)$ & 0.64 & 0.13 & $(0.15)$ & 0.39 & 0.01 & $(0.24)$ & 0.95 & 0.04 & $(0.09)$ & 0.70 \\
\hline Current smoking (yes vs no) & 0.19 & $(0.16)$ & 0.22 & 0.26 & $(0.16)$ & 0.09 & -0.03 & $(0.19)$ & 0.87 & 0.76 & $(0.30)$ & 0.01 & 0.22 & $(0.09)$ & 0.02 \\
\hline Fibr & 0.14 & & 0.56 & 0.26 & & 0.17 & 0.26 & & 0.23 & -0.61 & & 0.01 & 0.001 & & 0.99 \\
\hline Factor VIII activity $(\mid \mathrm{U} / \mathrm{ml}) \dagger$ & -0.32 & $(0.19)$ & 0.09 & -0.12 & $(0.13)$ & 0.33 & -0.05 & $(0.13)$ & 0.69 & 0.07 & $(0.17)$ & 0.69 & 0.09 & $(0.07)$ & 0.24 \\
\hline APTT & 0.00 & $(0.03)$ & 0.96 & -0.01 & $(0.03)$ & 0.67 & 0.04 & $(0.04)$ & 0.32 & 0.12 & $(0.05)$ & 0.02 & 0.03 & $(0.02)$ & 0.16 \\
\hline
\end{tabular}

Values are regression coefficients obtained with multivariate linear regression analysis with adjustment for age, gender, dementia, and symptomatic cardiovascular disease.

†Data on fibrinogen and APTT available for 245 subjects $(51,62,74,58)$; data on factor VIII activity for 734 subjects $(158,217,224,135)$.

Associations between APC response and cardiovascular risk factors differed by age, not gender. Overall, only smoking was associated with APC response: smokers had higher mean levels (table 2). In those aged 55-64 years inverse associations were found with total cholesterol $(0.14$ (95\% CI $0.01,0.27)$ decrease per $1 \mathrm{mmol} / \mathrm{l}$ increase) and diastolic blood pressure $(0.12(95 \% \mathrm{CI}-0.0,0.24)$ decrease per $10 \mathrm{mmHg}$ increase). In subjects aged $\geq 85$ years a strong positive association was seen with current smoking (0.76 (95\% CI 0.16,1.36) increase). Systolic pressure, HDL cholesterol and body mass index were not associated with APC response.

The associations between APC response and fibrinogen, factor VIII activity and APTT were different across the age strata (table 2). For fibrinogen, direction of the association in those aged 85 years or over was opposite to that of their younger counterparts. The association with factor VIII activity was generally inverse and most strong in those below the age of 75 years. The association with APTT was strongest in the very old.

\section{Discussion}

These findings suggest that in the elderly, age, gender, total cholesterol, diastolic blood pressure and smoking may influence APC response, the extent of which may differ by age.

APC response levels in our study were higher than reported by others. As a consequence, the prevalence of 'resistance to activated protein $\mathrm{C}^{\prime}$, was lower than those reported earlier. The relatively higher APC response levels are most likely due differences across studies in methods of processing the blood. We evaluated APC response in double centrifuged platelet poor plasma. A decreased number of platelets in plasma has been associated with an increased APC response. ${ }^{11}$ Alternatively, higher APC response levels in our cross-sectional study might in part be a consequence of survival, i.e., those with lower APC levels have either died or suffered from arterial disease, resulting 
in non-participation in the Rotterdam Study. When however, this notion is true, it is appears to be more apparent in women than in men (figure 1).

A low APC response has frequently been reported to be due to a single base mutation of the factor $\mathrm{V}$ gene. In some subjects, however, a low APC response was present in the absence of factor $\mathrm{V}$ Leiden mutation and some subjects with factor $\mathrm{V}$ Leiden mutation had a normal APC response. ${ }^{8}$ Furthermore, APC response is a continuous parameter which may yield valuable information concerning the protein $\mathrm{S} /$ protein $\mathrm{C}$ system across all levels. An unique feature of the present study is that APC response was looked upon as a continuous parameter, rather than a dichotomous one.

The main inference from the present study is that the level of APC response is to some extent determined by increasing age, female sex, increased diastolic blood pressure, increased total cholesterol and smoking. These factors should be allowed for in studies on the association between APC response and arterial disease. In particular when factors such as age, gender, diastolic blood pressure, cholesterol and smoking are differently distributed between cases and controls, unadjusted results may be biased towards a positive or negative finding.

\section{ACKNOWLEDGEMENTS}

The Rotterdam Study is supported in part a grant from the Netherlands Programme for Research on Ageing NESTOR (funded by the Ministry of Education, Cultural Affairs and Science and the Ministry of Health, Welfare and Sports), the Netherlands Heart Foundation, the Netherlands Organisation for Scientific Research (NWO), the Rotterdam Medical Research Foundation (ROMERES), and the Municipality of Rotterdam. The present study was in part supported by grant 92.398 from the Netherlands Heart Foundation.

We are grateful to the participants of the Rotterdam Study. We thank all field workers, and laboratory technicians in the Ommoord Research Centre and Bas Heijmans and Riet Kret from the Gaubius Laboratory for their enthusiasm and skilful contributions to the data collection.

\section{REFERENCES}

1. Dahlback B, Carlssn M, Svensson PJ. Familial thrombophilia due to a previously unrecognized mechanism characterized by poor anticoagulant response to activated protein C. Proc Natl Acad Sci 1993;90:1004-08.

2. Koster T, Rosendaal FR, Ronde de H, Briët E, Vandenbroucke JP, Bertina R. Venous thrombosis due to poor anticoagulant response to activated protein $\mathrm{C}$ : Leiden Thrombophilia Study. Lancet 1993;342:1503-6.

3. Halbmeyer WM, Haushofer A, Schön R, Ficher M. The prevalence of poor anticoagulant response to activated protein $\mathrm{C}$ among patients suffering from stroke or venous thrombosis and among healthy subjects. Blood Coagulation and Fibrinolysis 1994;5:51-7.

4. Bom JG van der, Bots ML, Haverkate F, et al. Reduced response to activated protein $C$ is associated with risk of stroke and transient ischemic attack. Ann Intern Med 1996 (in press)

5. Bertina RM, Koeleman BPC, Koster T, Rosendaal FR, Dirven $\mathrm{RJ}$, Ronde $\mathrm{H}$ de, et al. Mutation in blood coagulation factor $\mathrm{V}$ associated with resistance to activated protein C. Nature 1994;369:64-7.

6. Dahlback B, Hildebrand B. Inherited resistance to activated protein $\mathrm{C}$ is corrected by anticoagulant cofactor activity found to be a property of factor V. Proc Natl Acad Sci 1994;81:1396-400.

7. Voorberg J, Roelse J, Koopman R, Büller H, Berend F, Ten Cate JT, et al. Association of idiopathic venous thromboembolism with single point-mutation at Arg506 of factor V. Lancet 1994;343:1535-6.

8. Stirling D, White A, Clarkson J, Stewart A, Ludlam CA. A comparison between functional and genetic testing for activated protein $\mathrm{C}$ resistance. Blood Coag Fibrinol 1995;6:154.

9. Hofman A, Grobbee DE, De Jong PTVM, Vandenouweland FAM. Determinants of disease and disability in the elderly. The Rotterdam Elderly Study. Eur J Epidemiol 1991;7:40322.

10. Bom van der JG, Bots ML, de Bruijn AM, Hofman A, Grobbee DE. Measurement of B-thromboglobulin in the elderly. Findings from the Rotterdam Study. Fibrinolysis 1994;8(Suppl 2):157-9.

11. Sidelmann J, Gram J, Pederson OD, Jespersen J. Influence of plasma platelets on activated protein $\mathrm{C}$ resistance assay. Letter. Thromb Haemost 1995;74:990-7. 